\title{
BOTÁNICA Y NUMISMÁTICA: LAS PLANTAS EN LAS MONEDAS DE COSTA RICA (1709-2004)
}

\author{
José A. VARGAS-ZAMORA ${ }^{2,4}$ \& Jorge GÓmEZ-LAURITO ${ }^{2,3}$ \\ ${ }^{2}$ Escuela de Biología, Universidad de Costa Rica. 2060 San José, Costa Rica. \\ ${ }^{3}$ Investigador Asociado, Dept. Botany. Field Museum of Natural History, Chicago, Il., U.S.A. \\ ${ }^{4}$ Autor para correspondencia: javargas@biologia.ucr.ac.cr - Tel. (506) 207-3201
}

\begin{abstract}
The coins of Costa Rica include a variety of plant illustrations, ranging from a palm tree (Attalea rostrata ?) on the first known gold coin (1825), to a marine green algae (Caulerpa prolifera ?) on a silver commemorative piece of 1974. Cocoa (Theobroma cacao) seeds were legalized for use as currency in 1709. The national tree (Enterolobium cyclocarpum), and the national flower (Guarianthe skinneri) an orquid, were both represented in commemorative coins of 1975, and again the orchid in a silver piece of 1983. Coffee (Coffea arabica) and tobacco (Nicotiana tabacum), are represented as whole plants in coins of 1842 and 1847-1850, respectively. Coffee branches have been used since 1935 on most coins. The silk cotton tree (Ceiba pentandra), and an evergreen oak species (Quercus sp ?) are featured in mid XIX century pieces. Illustrations similar to the myrtle (Myrtus communis), and an unknown species of palm, were commonly used as wreaths in coins since 1842. Laurel (Laurus nobilis) is mentioned in decrees since 1863. However, desings more similar to M. cummunis than to L. nobilis were included in gold and silver coins.
\end{abstract}

Resumen: Las monedas de Costa Rica incluyen una variedad de ilustraciones de plantas, desde una palmera (Attalea rostrata ?) en la primera moneda de oro conocida (1825), hasta un alga marina (Caulerpa prolifera ?) en una pieza conmemorativa de 1974. Las semillas del cacao (Theobroma cacao) fueron utilizadas legalmente desde 1709 como moneda. El árbol nacional (Enterolobium cyclocarpum), y la flor nacional (Guarianthe skinneri), una orquídea, fueron ambas representadas en monedas conmemorativas de 1975 y la orquídea nuevamente en una pieza de plata de 1983. El café (Coffea arabica) y el tabaco (Nicotiana tabacum), están representadas como plantas completas en monedas de 1842 y 1847-1850, respectivamente. Las ramas de café han sido ilustradas en la mayoría de las monedas a partir de 1935. El árbol del algodón sedoso (Ceiba pentandra) y una especie siempre verde de encina (Quercus sp ?) han sido grabadas en piezas de mediados del siglo XIX. Ilustraciones similares al mirto (Myrtus communis) y a una especie desconocida de palma, fueron incluidas en coronas en las monedas desde 1842. El laurel (Laurus nobilis) es mencionado en decretos desde 1863. Sin embargo, diseños más similares a M. communis que a L. nobilis fueron incluidos en monedas de oro y plata.

Palabras clave / Key words: numismatics, coins, Attalea, Caulerpa, Ceiba, Coffea, Enterolobium, Guarianthe, Laurus, Myrtus, Nicotiana, Quercus, Theobroma, Costa Rica.

Durante la última década se ha dado en Costa Rica un resurgimiento del interés en el estudio de las monedas, medallas, billetes y boletos de café. Este auge ha sido motivado, entre otras razones, por la apertura del Museo de Numismática y la publicación de libros y folletos, como los de Dueñas Leiva (1994), Gurdián Montealegre (1997), Chacón (2000), Carranza Astúa

\footnotetext{
${ }^{1}$ Conferencia impartida en el auditorio del Museo de Numismática, Plaza de la Cultura, San José, Costa Rica, 27 de mayo, 2004.
}

(2001), Chacón-Hidalgo y Carazo de Flores (2002), Chacón Hidalgo (2003) y Murillo (2004), así como por la serie de conferencias patrocinadas por el Museo. Esta conferencia es parte de esta serie.

Desde hace algún tiempo se nos ha consultado sobre los nombres científicos de algunas especies de plantas representadas en monedas de Costa Rica. Si bien en los decretos del siglo XIX y en algunos del siglo XX la información no es tan detallada, hemos decidido escribir ésta nota y aportar nuestro criterio. Esperamos que ésta avive aún más el interés en la 
numismática, especialmente entre los jóvenes que no tuvieron, por motivos de la devaluación del colón, la oportunidad de comprar una melcocha de Cocos nucifera de a dos reales, pero que todavía emplean las palabras pesos y plata en el quehacer diario.

La palabra moneda tiene su origen en el latín Moneta, nombre dado a la diosa Juno, guardiana de las memorias y de los archivos, en cuyo templo se construyó el taller de acuñación (Blanco Quirós 1944). El lector interesado puede consultar el libro de León y Poveda (2000) y los catálogos de Krause y Mishler $(2001,2004)$ para información sobre las plantas y las monedas de Costa Rica, respectivamente. En las figuras utilizadas en esta conferencia se indica al pie el valor de la moneda, el metal, el país o lugar donde se le acuñó, el año de acuñación, su diámetro en milímetros $(\mathrm{mm})$ y peso en gramos $(\mathrm{g})$, según Gurdián Montealegre (1997). Algunas monedas se ilustran con su diámetro real, en otras este ha sido reducido o aumentado.

\section{EL CACAO}

Desde el comienzo del siglo XVI hasta 1865 se utilizaron en Costa Rica monedas de oro y plata según el sistema de España. Las monedas de plata (Real) y las de oro (Escudo, Onza) guardaban una relación de 16 a 1, esto es: 16 reales $=1$ Escudo $=2$ pesos, 16 pesos $=1$ Onza (Chacón Hidalgo 2003). De ahí proviene la expresión popular no afloje ni un 16, utilizada hasta hace un tiempo para indicar el no ceder lo mínimo, sea en dinero o en esfuerzo. A la moneda de 8 reales se le conocía como Peso. El escaso comercio de la época hacía que las monedas fueran difíciles de obtener y el cacao (Theobroma cacao, Sterculiaceae, Fig. 1), era un medio frecuente de pago en Mesoamérica. Pedro de Anglería (1994) dice sobre ella: Oh feliz moneda que da al humano linaje una bebida suave y útil y a sus poseedores los libra de la tartárea peste de la avaricia, porque no se la puede enterrar ni guardar mucho tiempo. Esta escasez en la Costa Rica del siglo XVIII provocó que a partir de 1709 las semillas o almendras del cacao se utilizaran legalmente, por decreto del gobernador don Lorenzo Granda Balbín, como dinero, de modo que todos los vecinos, criadores de ganados, los que vendieren candelas, maiz, dulce, sal, manteca, jabón, sebo y demás cosas tocantes al mante-
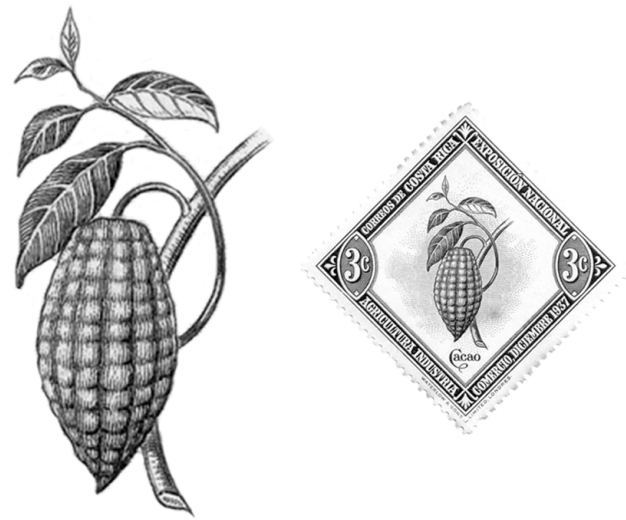

Figura 1. Fruto y hojas del árbol de cacao ilustrados en la estampilla de correos de Costa Rica, 1937, valor 3 céntimos, impresa con tinta pardo oscuro por Waterlow and Sons, Londres, $36 \mathrm{~mm} \times 36 \mathrm{~mm}$. Colección privada.
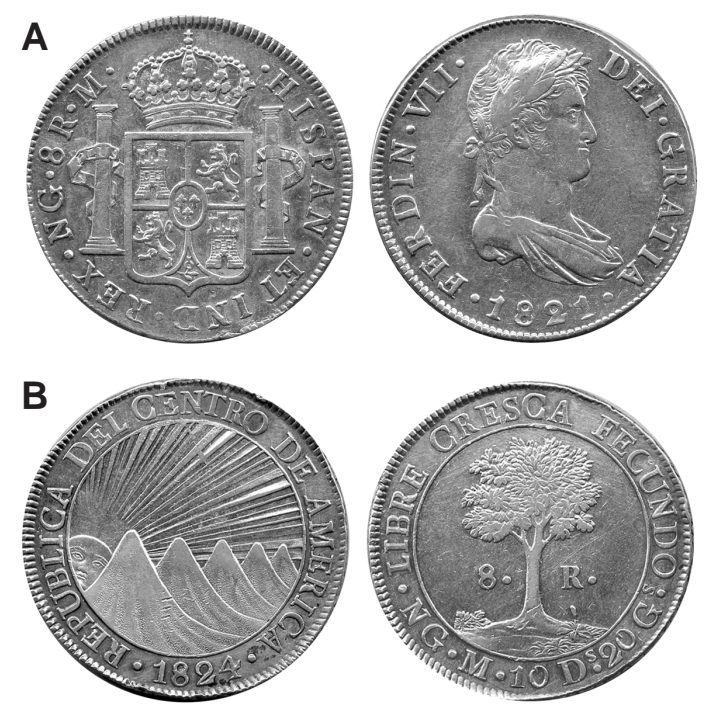

Figura 2. Anverso y reverso de: A. Ocho reales (8R), plata, Guatemala, 1821, $39 \mathrm{~mm} / 27$ g. Efigie del rey Fernando VII. Colección privada. B. Ocho reales (8R), plata, Guatemala, 1824, 39 mm / 27.07 g. Colección privada.

nimiento, reciban por ello cacao siendo bueno dar y recibir, pena de diez pesos a los dichos criadores y de cinco pesos a los que venden los demás géneros mencionados (Valerio 1962). Las semillas de cacao son resistentes, fáciles de transportar y contar, pero difíciles de falsificar; sin embargo, algunos indígenas lo hacían rellenando con barro el interior de la semilla (Fernández de Oviedo 1944). Los indígenas cosechaban el cacao desde tiempos prehispánicos. Sin embar- 
go, las primeras plantaciones pertenecientes a ciudadanos de Cartago datan de principios del siglo XVII. En 1710 la tasa de cambio era de 100 semillas por un real (Valerio 1962) y un siglo después según González Zeledón (1998) el precio era de ocho manos (o sea cuarenta almendras) por un real de los de las reales armas de don Fernando VII, que era la base de la moneda circulante. La Fig. 2A ilustra el anverso y reverso de una moneda de 8 reales (8R), de Fernando VII acuñada en Nueva Guatemala (NG). Sobre el cacao no estamos seguros si las almendras usadas como monedas terminaban de inmediato en chocolate, o si por pasar de mano en mano estaban al cabo muy devaluadas para ser transformadas en el alimento de los dioses $(=$ Theobroma). Según lo indica Valerio (1962), es posible que el tostado del grano eliminara toda posibilidad de infección. En las reuniones sociales o de negocios el chocolate se tomaba en una jícara (fruto seco del árbol Crescentia cujete, Bignoniaceae), sin dulce, tibio y muy espeso; de ahí el refrán, cuentas claras-chocolate espeso.

\section{LA PALMERA}

En 1824 Costa Rica ingresó en el sistema federal como una de las Provincias Unidas del Centro de América. Cada estado miembro debía acuñar su propia moneda de acuerdo con el decreto federal emitido en Guatemala ese año, el cual indicaba que el reverso de las monedas debía presentar un árbol como emblema de libertad (Gurdián Montealegre 1997). La Fig. 2B ilustra el anverso y el reverso de la moneda de 8 reales acuñada en Guatemala en 1824 de acuerdo con esa normativa. Sobre la especie de árbol ilustrada escribimos más adelante. Para los ciudadanos de la época debió ser un gran cambio y sensación de libertad el pasar de las monedas con el busto del rey y el escudo de España (Fig. 2A), a otras con un árbol y una cadena de volcanes (Fig. 2B). Sin embargo las primeras monedas de Costa Rica, de las cuales se conservan solo tres ejemplares, son las de medio escudo (= un peso) de 1825, que incluían una palmera (Fig. 3). El 10 de mayo de 1823 se promulgó el primer decreto de la Provincia de Costa Rica para el establecimiento de un cuño y la troquelación de su propia moneda (Lines 1946, Murillo 2004). Este decreto, que no fue ejecutado por falta de recursos, indicaba que la moneda lle-
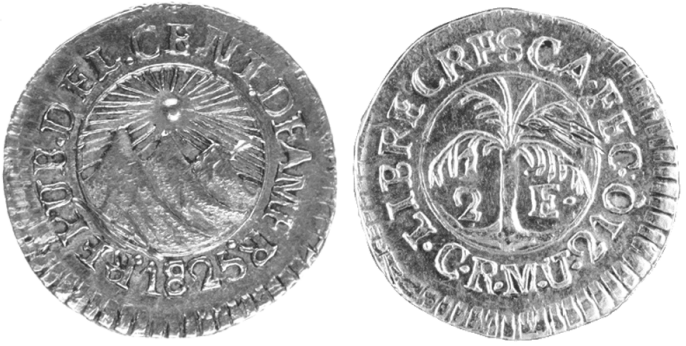

Figura 3. Anverso y reverso del medio escudo $(1 / 2 \mathrm{E})$. Oro. Los Horcones. 1825. $16 \mathrm{~mm} / 1.8$ g. Museo Numismática BCCR.

varía en el reverso una palma en el centro cruzada por una espada y un fusil con bayoneta y cañón por debajo (Lines 1946). Don Mateo Urandurraga (MU, Fig. 3) extrajo oro de la mina Los Oreamunos en los Montes del Aguacate y acuñó esas monedas en el ingenio de metales San José de Los Horcones, a orillas del Río Grande y como a $6 \mathrm{~km}$ de la mina (Melliss 1891). Del ingenio aún hoy existen algunas reliquias en el sitio original (Murillo 2004). El uso de la palmera, en vez de un árbol, desató una reacción negativa sobre la aceptación estatal y federal de la nueva moneda, provocando que la Comisión de Hacienda de Costa Rica argumentara que...en la moneda de Guatemala aparece un árbol copado arriba, su vástago derecho y sin ramas y en la nuestra se advierte una palma,... notable diferencia que hará que la nuestra no tenga crédito. Don Juan Mora Fernández respondió indignado que...el Gobierno ignora que haya hasta ahora naturalista alguno negado a la palmera la propiedad y cualidades de árbol (Gurdían Montealegre 1997). Cabe mencionar que el término árbol define al vegetal leñoso, por lo menos de 5 metros de altura, con tallo simple (en este caso denominado tronco) hasta la llamada cruz, en que se ramifica y forma la copa (Font Quer 1973), por lo que don Juan estaba en lo correcto. El explorador Thomas F. Meagher en su viaje desde Puntarenas a San José en 1858 dice de las selvas de La Chacarita: alli en todas sus variedades teníamos la palmera, esa princesa del reino vegetal como la llamó Linneo (Fernández Guardia 2002). La vegetación de los Montes del Aguacate también debió ser exuberante en esas épocas y suponemos que la palmera Attalea rostrata (Arecaceae), palma real o corozo (Hammel et al. 2003), cuyas frondas proveían el principal material para techar casas y ranchos, era una es- 
pecie con la cual la mayoría de los pobladores estaban familiarizados. A. rostrata crece hasta 20 metros de altura y algunos ejemplares aún sobreviven en los deforestados Montes del Aguacate. Su forma, abundancia, utilidad práctica y el crecer libre entre los árboles, tal vez fueron las razones que pesaron en su escogencia para la moneda (Fig. 3), si ese fue en realidad el modelo. El nombre A. butyracea (León y Poveda 2000) se considera sinónimo de $A$. rostrata. Hemos descartado a otras especies de la zona como la pacaya (Chamaedorea costaricana) por tener tallos múltiples de poco grosor, al coyol (Acrocomia aculeata) y al pejibaye (Bactris gasipaes) por presentar largas espinas en sus troncos. El cocotero (Cocos nucifera) era más abundante en la costa Caribe y las pipas no aparecen en la moneda. En la antigüedad las palmeras simbolizaban permanencia por ser muy longevas y sus hojas (palmas) representaban a la paz.

\section{LA CEIBA}

En 1828, por decreto de don Juan Mora, se inició la acuñación de monedas de oro de $8,4,2,1$ y $1 / 2$ escudos. En 1832 (con troqueles fechados 1831) empezó la producción de piezas de plata de $8,2,1$, y $1 / 2$ reales (Murillo 2004). Estas acuñaciones se hicieron siguiendo la directriz federal de incluir, como emblema de libertad, un árbol como el del peso de 1824 (Fig. 2B). La Fig. 4A ilustra un ejemplar de un real (1R) de Costa Rica (CR), 1831, el cual representa una de las primeras monedas del país con el árbol requerido, así como en el escudo de oro de 1842 (Fig. 4B). En 1845, por decreto de don José Rafael de Gallegos se autorizó la circulación de pesetas españolas en su equivalente de dos reales, mediante resellos de un árbol en el anverso (Fig. 4C) y de un busto de mujer en el reverso. Por ésta razón esas monedas reselladas, así como las equivalentes posteriores de $1 / 4$ de peso, 25 centavos y 25 céntimos se conocieron como pesetas hasta finales del siglo XX. En 1845 también se acuñaron las únicas monedas de Costa Rica de un cuarto de real, que incluyen un árbol (Fig. 4D). En 1846 se resellaron, también con un árbol (Fig. 4E), las monedas macuquinas (= moneda colonial hispanoamericana de bordes recortados y espesor variable) que circulaban en la región. El árbol podría ser asignado a la especie Ceiba pentandra (Bombaca-
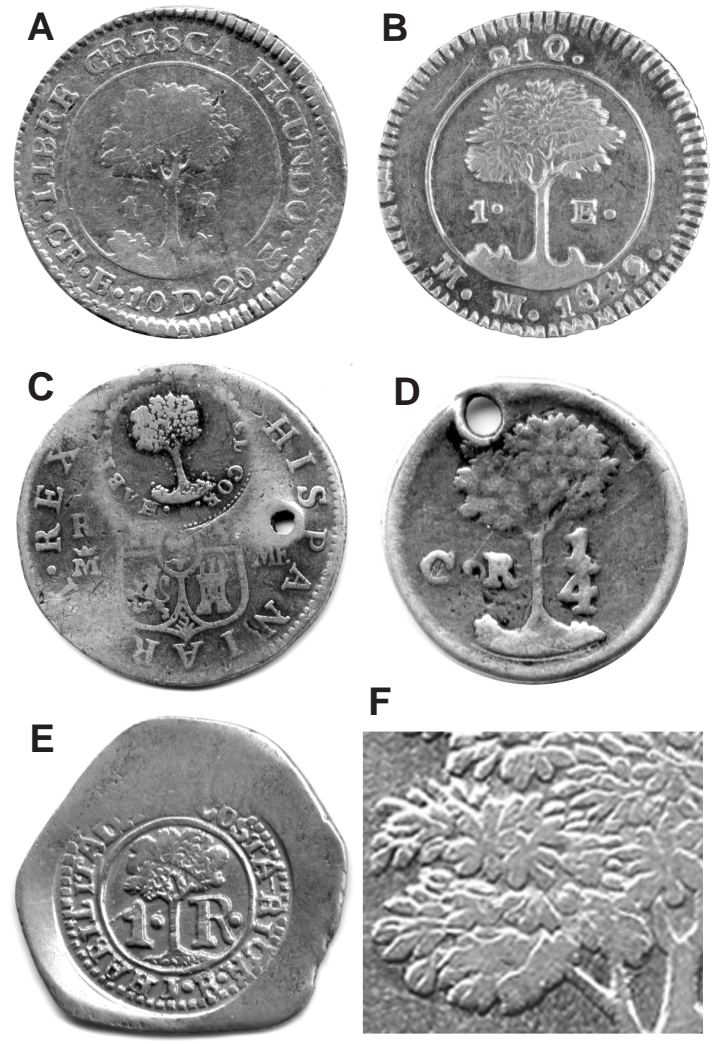

Figura 4. A. Un real (1R), plata, San José, 1831, 20 mm / 3.4 g. Colección privada. B. Un escudo (1E), oro, San José, 1842, 19 mm / 3.0 g. Museo de Numismatica BCCR. C. Resello (1845), sobre peseta española de 2 reales, 1799. Colección privada D. Un cuarto de real, plata, San José, $1845,11 \mathrm{~mm} / 0.8$ g. E. Resello de 1 real (1846) sobre moneda macuquina de plata. Colección privada. F. Detalle de una de las ramas de la ceiba de la moneda de 8 R, 1824, (Fig. 2 B). Nótese las hojas propias de la especie C. pentandra.

ceae), ceiba o ceibo, símbolo de vida para la población Maya-Quiché y refugio de los espíritus para los indios guatusos de Costa Rica. Es el Árbol Nacional de Guatemala desde 1955 (Rojas 1993). Las autoridades españolas plantaban la ceiba en el centro de las plazas públicas (Murillo 2004). C. pentandra es un árbol grande, que crece hasta más de 30 metros de altura, con tronco recto, cilíndrico, grueso y abultado en su parte media, como es típico de éstas bombacáceas. Sin embargo, en las monedas aparece un árbol joven, con tronco delgado y gambas apenas visibles en su base, representando así al joven gobierno federal y al cual se le augura Libre Crezca Fecundo. La C. pen- 
tandra posee hojas digitadamente compuestas con 5 a 9 hojuelas, que el grabador trató de reproducir en la moneda (Fig. 4F). La abundancia de esta especie en los Montes del Aguacate fue también mencionada por T. Meagher así: ... allí estaba la ceiba o árbol de algodón sedoso, cuyo tronco alcanza tales dimensiones que en él se cavan las canoas más grandes (Fernández Guardia 2002). Ceiba es también el nombre de uno de los distritos del cantón de Orotina. En 1970, por iniciativa del gobierno de don José J. Trejos se acuñó una serie de monedas de oro y plata conmemorativas de los 150 años de la Independencia. En la moneda de plata de 10 colones, que en su reverso se asemeja en varios aspectos a la de 1824 (Fig. 2B), se ilustra nuevamente esta ceiba. En marzo de 1963 fue plantada una ceiba en el jardín de la Casa Amarilla (San José). Este árbol es actualmente un gigante y en los años 1972-1974 se incluyó su dibujo en el reverso del billete de 50 colones, Serie C, del BCCR (Carranza Astúa 2002).

\section{EL TABACO Y EL CAFÉ}

Don Braulio Carrillo decretó en 1840 que sus nuevas monedas representen al reverso un árbol que figure al del café en el oro y al tabaco en la plata (Gurdían Montealegre 1996). Sin embargo, en las monedas de oro resultantes (1 escudo, 1842, Fig. 4B) el árbol ilustrado es una ceiba semejante a la de la emisión de 1831. Según indica Fernández Guardia (1980) la falta de troqueles apropiados no permitió acuñar moneda con el árbol del café y se siguió empleando el de la Federación. La única moneda de plata emitida es la de $1 / 2$ real, 1842 , y en ésta se grabó una planta de tabaco (Nicotiana tabacum, Solanaceae) reconocible por su forma, hojas grandes sin pecíolo y con la base más o menos envolvente (Fig. 5A). En éstas monedas de don Braulio también aparecen por primera vez, bajo la estrella, las dos ramas entrelazadas de dos especies diferentes de plantas (Fig. 10A), de cuya identidad escribimos al final de ésta nota. El comercio del tabaco era una de las pocas actividades lucrativas de principios del siglo XIX y era monopolio del Estado, ejercido a través de la Factoría de Tabacos. Los indígenas inventaron el fumado desde tiempos prehispánicos. Sin embargo, los primeros cultivos documentados datan de 1632 y para 1781 la Factoría llegó a ser
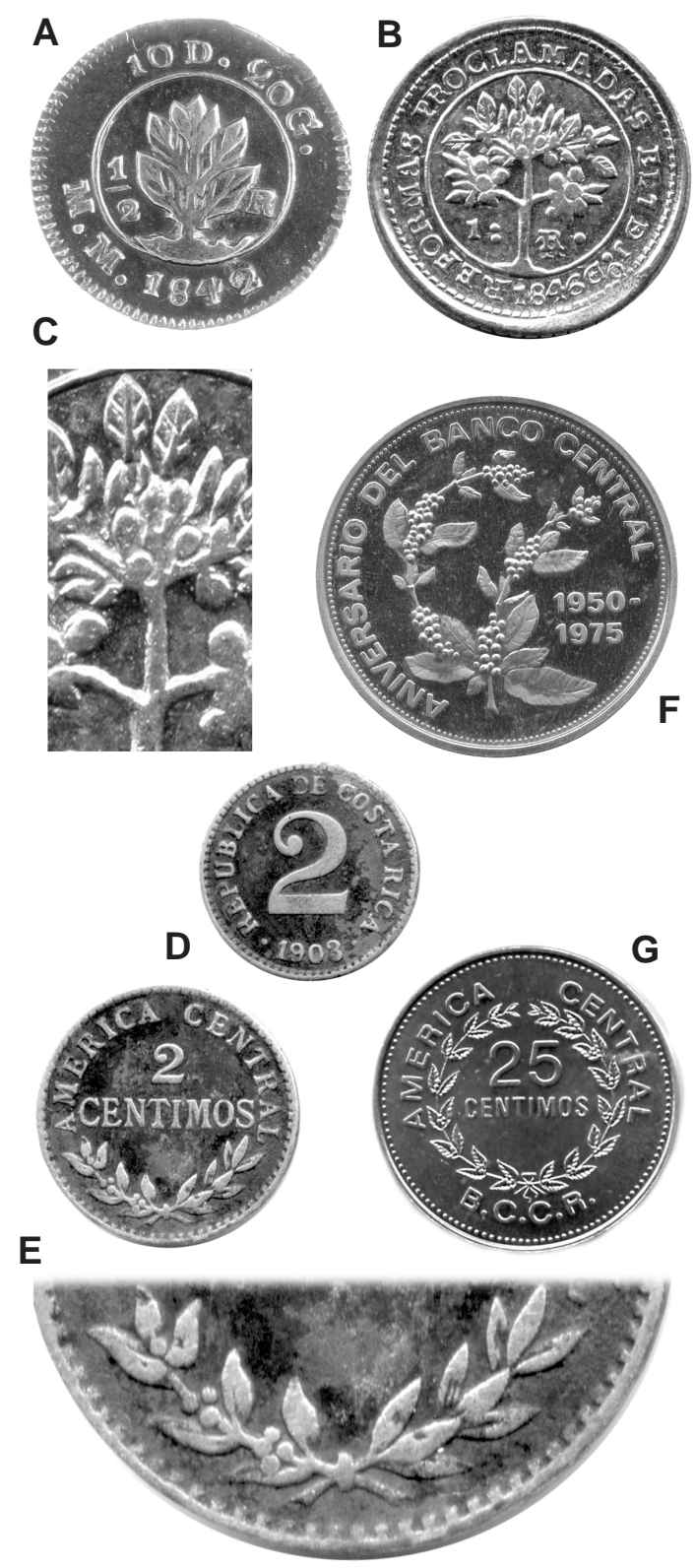

Figura 5. A. Medio real, plata, San José, 1842, $18 \mathrm{~mm} /$ 1.5 g. Museo de Numismática BCCR. B. Un real, plata, San José, 1847, 20 mm / 2.9 g. Colección privada. C. Detalle de la moneda de un real, mostrando hojas de café y sus nervaduras. D. Anverso y reverso (ampliado) de la moneda de dos céntimos, níquel, Estados Unidos, 1903, 15 mm / 1 g. Bandola de café y mirto. Colección privada. E. Detalle de la moneda de 2 céntimos. Nótese las formas diferentes de las hojas del café y del mirto. F. Cinco colones, níquel, Alemania, 1975. $30 \mathrm{~mm} / 12 \mathrm{~g}$. Colección privada. G. Veinticinco céntimos, aluminio, Canadá, 1982, 23 mm / 3.4 g. Bandolas de café. Colección privada. 
la primera institución económica de la colonia. En 1821 había en su bodega 160.000 libras ( 1 libra $=16$ onzas) de tabaco, el cual se vendía a cuatro onzas por un real (Fallas Barrantes 1967). En agosto de 1836, debido a una situación de emergencia y necesidad de generar recursos, se decretó la venta forzosa de tabaco mediante su adjudicación a los habitantes con capacidad de hacerlo (Villalobos Rodríguez et al 2000). El reciente descubrimiento de cinco vales de tabaco de 1836 , hasta por 250 pesos, mucha plata para esa época, enfatiza la importancia de esta planta en la economía del país y justificaba su inclusión en una moneda. Estos vales podrían haber sido utilizados como pre-billetes (Green 2003), tal vez porque en este caso particular era un poco incómodo llevar en el bolsillo 250 monedas (similares a las de la Fig. 2B) cuyo peso total era 6767 gramos (g). Si por el contrario, el mismo monto fuese acarreado en su equivalente de oro en monedas de 4 escudos, habría que llevar 31 de ellas, más una de 1 escudo, para un total de $422 \mathrm{~g}$, lo cual posiblemente era más cómodo, pero riesgoso aún en el San José de 1836. No es casualidad que los $422 \mathrm{~g}$ en piezas de oro equivalen a $1 / 16$ de los $6767 \mathrm{~g}$ de plata.

La planta de café (Coffea arabica, Rubiaceae), que se debió incluir en la moneda de 1842, esperó hasta 1847 cuando, por circular de don José María Alfaro, se emitieron las primeras monedas (1 real) conmemorativas en Costa Rica (Fig. 5B) cuyo tipo sea por el anverso un árbol de café ocupando el centro (Gurdián Montealegre 1997). Un total de 2200 de esas monedas de plata fueron botadas (lanzadas) al pueblo en ceremonias especiales (Murillo 2004). De ahí tal vez proviene la expresión local botar la plata. Es importante mencionar que en 1847 los cafetales estaban sembrados de plantas muy diferentes a las variedades actuales de pequeña altura y alta producción (Coste 1968). Esas primeros cafetos de C. arabica alcanzaban más de 5 metros de altura, tanto así que aún entrado el siglo XX la cogida en esas plantaciones se hacía utilizando escaleras y el fruto se recogía en manteados colocados en el suelo bajo los árboles. Una escena similar a la descrita se ilustra en el billete de 10 colones, Serie C, del BICR (Carranza Astúa 2002). El arbusto de café está bien ilustrado en la moneda, con sus ramas (bandolas) opuestas y frutos en racimos (llamados copetes), en los nudos de las hojas opuestas y de forma ovalada. Aún más, las nervaduras dibujadas (Fig. 5C) se asemejan a las de las hojas de las plantas reales. Estas monedas de un real, así como las acuñadas en 1849 y 1850 se conocen como mariquitas, siendo en las dos últimas donde por primera vez aparece el nombre República de Costa Rica (Chacón Hidalgo 2002).

Por incluir al café, hacemos aquí a modo de paréntesis este comentario sobre monedas del siglo XX: no fue sino hasta 1903 que en la moneda de dos céntimos (Fig. $5 \mathrm{D}, \mathrm{E})$ se representó, a la izquierda, una rama semejante a una bandola de café. Las bandolas, algunas veces muy modificadas, han sido grabadas en casi todas las monedas de uso común en Costa Rica desde 1935. En 1975, durante el gobierno de don Daniel Oduber, el café fue nuevamente motivo para una moneda conmemorativa. Esta vez fue en la pieza de níquel de un valor de 5 colones que incluye dos bandolas con frutos (Fig. 5F). Entre las monedas de uso corriente se incluye el reverso (Fig. 5G) de una moneda de 25 céntimos de 1982, con dos bandolas de C. arabica.

\section{LA ENCINA}

En 1848, durante la administración del Dr. Castro se emitió el decreto que inició la denominación de $P e$ so $\left(\mathrm{P}^{\mathrm{O}}\right)$ en las monedas de plata, si bien solo se acuñaron en valores de $1 / 4,1 / 8$ (Fig. 6A) y $1 / 16$ de peso (Gurdián Montealegre 1997). También se acuñó, con troqueles traídos de Inglaterra (Murillo 2004), monedas de oro de $1 / 2$ onza y de 2,1, y $1 / 2$ escudos, conocidas como de la India Parada, por la figura de pie que tiene poco de india y mucho de arquera olímpica (Fig. 6B). La India Parada es una moneda elegante, que refleja la adopción de valores y cánones estéticos europeos propiciada a través del auge comercial promovido por el cultivo del café (Chacón Hidalgo 2003). Era el metal común en el bolsín de redecilla de pita o seda relleno de onzas, medias onzas, cuartas, escudos (González Zeledón 1998) de la clase pudiente. La otra, la de plata, era una moneda de diseño innovador, con otra especie de árbol ocupando casi todo el reverso (Fig. 6A,C). Sería la plata que llevaba anudada en el pañuelo la gente del pueblo que se reunía los sábados en la Plaza Principal (hoy Parque Central) a comprar los víveres de la semana. En el San José de 1858 el que tenía recursos podía vestir un pantalón de casimir inglés de calidad superior de a 12 pesos y botas de 


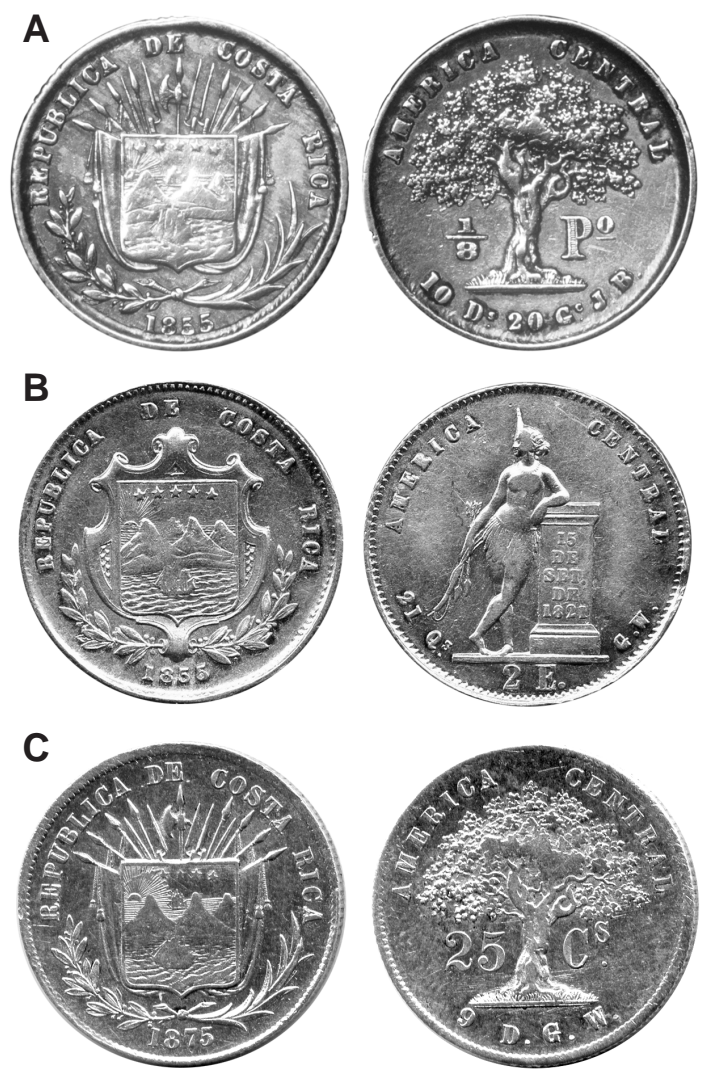

Figura 6. Anverso y reverso de: A. Un octavo de Peso $\left(\mathrm{P}^{\mathrm{o}}\right)$, plata, San José, 1855, $19 \mathrm{~mm} / 2.9 \mathrm{~g}$. Museo de Numismática BCCR. B. Dos escudos (2E), oro, San José, 1855, $23 \mathrm{~mm} / 6.7 \mathrm{~g}$. Colección privada. C. Veinticinco centavos $\left(25 \mathrm{C}^{\mathrm{S}}\right)$, San José, 1875, $24 \mathrm{~mm} / 6.25 \mathrm{~g}$. Colección privada.

charol de 8 pesos, mientras que el salario promedio de un jornalero por mes oscilaba entre 15 y 18 pesos y la zaraza, que era la tela de vestir de la mujer descalza del jornalero, se vendía a un real la vara (Vega Jiménez 1991). El decreto de 1848 especifica que la moneda de plata contendrá un árbol de encina sobre un terreno figurado (Lines 1948). Las encinas (Quercus spp, Fagaceae), por su gran tamaño y longevidad, han tenido gran importancia en varias culturas desde tiempos bíblicos. A estos árboles se les ha considerado símbolo de hospitalidad, así como también se les asocia con el dios del trueno en algunos pueblos europeos. Un ramo de hojas de encina es símbolo de heroísmo y victoria. Su madera dura es símbolo de fortaleza y vida eterna. En Inglaterra los primeros reyes consideraban a los encinares como símbolo de rique- za y como un criterio para calcular impuestos. En América del Norte, la madera de Q. virginiana era el material más buscado para la construcción de buques a mediados del siglo XIX (Lust 1990, Ciesla 2002). La encina familiar para los españoles que venían a radicar en Costa Rica fue $Q$. ilex, especie abundante en la península Ibérica, donde crece hasta 12 metros con tronco grande ramificado en varios brazos y copa redonda. Para dibujar una encina el grabador, posiblemente don Manuel Castro, quien en esos años fue enviado a Inglaterra a instruirse en los oficios de grabador y ensayador (Murillo 2004), tal vez consultó un libro de botánica o se asesoró con un naturalista visitante para localizar una especie similar en el país, como $Q$. costaricensis ó $Q$. seemannii, en las vecindades de San José, o grabó una especie inglesa de Quercus durante su estadía en Europa. Sobre el árbol en la moneda lo que podemos decir es que se asemeja mucho a un Quercus, sin precisar la especie. Esta familia de árboles incluye doce especies en Costa Rica (Burger 1977). Es importante mencionar que el científico danés Anders Oersted estuvo de visita en San José en 1847. Las visitas de naturalistas no pasaban desapercibidas para los notables de la época, como los alemanes Johan Barth y Wilhem Witting ensayadores de la Casa de Moneda (Fernández Guardia 2002), cuyas iniciales JB (Juan Barth) y GW (Guillermo Witting) aparecen en monedas de la época (Figs. 6A,B,C).

En 1863, durante la administración de don Jesús Jiménez, se decretó la adopción del sistema decimal $(100$ centavos $=1$ Peso $)$. En la moneda de plata se continuó utilizando un árbol de encina sobre un campo figurado. Las últimas monedas con la encina se acuñaron en 1875 (Fig. 6C). En Costa Rica a las monedas con el Quercus se les conoce como de arbolito (Murillo 2004).

\section{EL ARBOL DE GUANACASTE}

El 1975 se emitió la moneda conmemorativa de 10 colones con un árbol de guanacaste en el reverso. Esta especie fue declarada Arbol Nacional de Costa Rica por decreto del 31 de Agosto de 1959. El árbol Enterolobium cyclocarpum (Fabaceae) se le encuentra desde México hasta el norte de Sudamerica y desde el nivel del mar hasta los 1000 metros. Crece hasta $50 \mathrm{~m}$ de altura con tronco robusto y ramas gruesas, largas, 

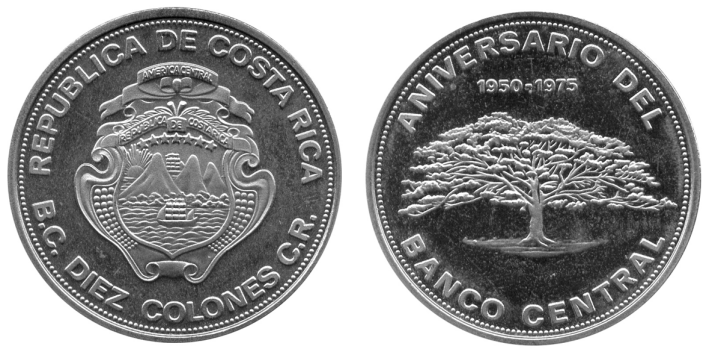

Figura 7. Anverso y reverso de la moneda de diez colones, níquel, Alemania, 1975, 33 mm / 16 g. Colección privada.

visibles a través del follaje como en el grabado de la moneda (Fig. 7). Sus flores blancas y frutos como orejas son apetecidos por el ganado. Es símbolo de estabilidad y crecimiento (Altamirano 1997).

En algunos casos se le confunde con la especie de chilamate Ficus goldmanni (Moraceae), como el ilustrado en una estampilla de correos de Costa Rica (20 céntimos, 1969). El higuerón, $F$. goldmanni también es común en Guanacaste y su copa muy densa en forma de sombrilla, con ramas casi equidistantes del suelo, lo hacen refugio ideal contra el calor de la pampa. Como bien lo apunta Lines (1946), el árbol de la ceiba se utilizó a partir de 1924 en el escudo del Banco Nacional de Seguros, si bien el reglamento original del Banco dice erróneamente que se usará el dibujo del árbol de Guanacaste que tenía la moneda de la federación centroamericana.

\section{LA GUARIA MORADA}

La Flor Nacional de Costa Rica (Decreto del 15 de junio de 1939) ha sido incluida en dos monedas conmemorativas. La primera (Fig. 8A) en 1975 (Decreto
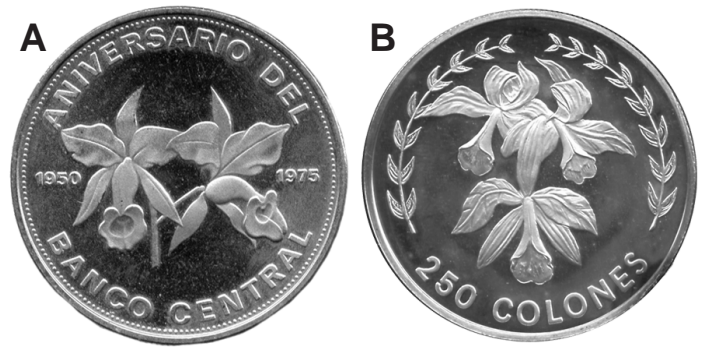

Figura 8. Reversos de: A. Veinte colones, níquel, Alemania, 1975, 36 mm / 20 g. Colección privada. B. Doscientos cincuenta colones, plata, Estados Unidos, 1983, 39 mm / $30.3 \mathrm{~g}$. Colección privada. de don Daniel Oduber) y la segunda (Fig. 8B) en 1983, por decreto de don Luis A. Monge. Recientemente, Cattleya skinneri (Orchidaceae) fue asignada a un nuevo género (Guarianthe), de modo que su nombre correcto es Guarianthe skinneri. La asignación al nuevo género fue el resultado de estudios del ADN (Acido DesoxirriboNucleico) del grupo Cattleya y se deriva directamente del término costarricense para designar a las orquídeas como guarias (Dressler \& Higgins 2003). En algunas regiones de Costa Rica los labriegos creen que la guaria morada atrae la dicha y la buena suerte. Además, se dice que confiere unión y concordia familiar (Altamirano 1997). Esta orquídea está bien ilustrada en ambas monedas. Las ramas que rodean a las guarias en la Fig. 8B son de café, aunque aquí las hojas aparecen sésiles y con nervación modificada.

La guaria morada también fue incluída en el anverso de los billetes de 5 colones, Serie D, del BCCR, emitidos desde 1968 hasta 1992 y considerados por los coleccionistas como uno de los billetes más bellos del mundo (Carranza Astúa 2002). De la obra Manojo de Guarias (Chavarría 2003) del poeta naturalista costarricense Lisímaco Chavarría (1878-1913), hemos seleccionado estos versos sobre la guaria morada, la ceiba y las encinas:

... caprichos de amatista suspendidos en los troncos de ceibas centenarias, fulgores de la aurora detenidos sobre el remanso azul, así las guarias.

... recibe este manojo hecho de guarias que fueron el collar de las encinas; ellas te llevan las cadencias varias que saben las dulzainas campesinas.

\section{EL ALGA MARINA}

En las monedas conmemorativas emitidas en 1974 por decreto de don José Figueres, se incluye, en la pieza de 50 colones, dos tortugas verdes (Chelonia mydas) sobre un fondo de agua que contiene la figura de una planta (Fig. 9A). En la moneda de 100 colones de ese año aparece la misma planta (Fig. 9B) bajo un manatí (Trichechus manatus). Frecuentemente estas ilustraciones de temas marinos incluyen a Thalassia testudinum (Hydrocharitaceae) que son plantas su- 

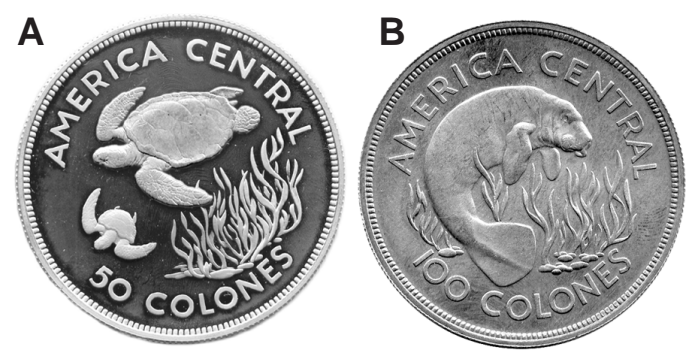

FIGURA 9. Reversos de: A. Cincuenta colones, aleación, Inglaterra, 1974, $38.6 \mathrm{~mm} / 25.3 \mathrm{~g}$. Colección privada. B. Cien colones, aleación, Inglaterra, 1974, 42 mm / 31.6 g. Colección privada.

mergidas, erectas, con hojas largas, verdes, individuales, de unos $10 \mathrm{~mm}$ de diámetro, ápice romo y con $9 \mathrm{a}$ 15 venas paralelas.

Sin embargo, en este caso el grabado de la planta posee hojas terminadas en punta, sin venas y ramificadas. Nosotros especulamos que la especie más afín a la grabada es una forma del alga verde (Chlorophyta) Caulerpa prolifera (Caulerpaceae). En la costa Caribe de Costa Rica C. prolifera se encuentra abundante en Punta Uva, sobre fondos arenosos o en lechos de T. testudinum, hasta unos $15 \mathrm{~m}$ de profundidad (Soto y Ballantine 1986).

\section{SOBRE MIRTOS, PALMAS Y LAURELES}

Desde hace miles de años algunas especies de plantas, como el mirto, el laurel y las palmas, han sido utilizadas en ceremonias asociadas a situaciones de triunfo o mérito. El mirto fue consagrado a la diosa Venus y con sus ramas se coronaba a los magistrados atenienses. El laurel fue consagrado al dios Apolo y su corona se usaba como símbolo de victoria, llamándose laureados a los así coronados. Las palmas se utilizaban también como símbolo de victoria, de alegría, o de permanencia, por ser la palmera una planta que vive por muchos años. Estas plantas aparecen usualmente en el reverso de las monedas de Costa Rica, siendo la primera vez en las acuñadas en el gobierno de don Braulio Carrillo, que incluyen dos ramas semejantes a una palma y al mirto (Figs. 10A). La inclusión de esas ramas podría haber sido para ser congruente con el formato del Escudo del Estado de Costa Rica decretado en 1840 por don Braulio (Villalobos Rodríguez et al 2000). La rama incluída junto al mirto en varias monedas (Figs. 10,11) se asemeja a
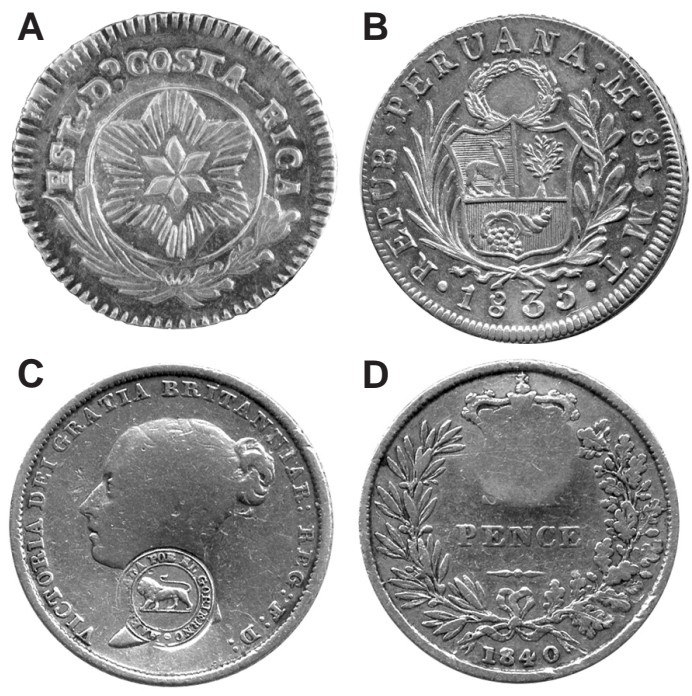

Figura 10. A. Anverso de un escudo, oro, San José, 1842, 19 mm / 3.0 g. Colección Museo de Numismática BCCR. En la Fig. 4B se ilustra el reverso de este escudo. B. Anverso de la moneda de 8 Reales, Perú, 1835, $39 \mathrm{~mm} /$ 26.6g. Colección privada. Anverso (C) y reverso (D) de la moneda inglesa de seis peniques (six pence), plata, 1840, con resello circular de $6 \mathrm{~mm}$ de ancho con el león pasante en el centro y leyenda Habilitada por el Gobierno (de Costa Rica) en la orla. Moneda equivalente a un real en Costa Rica. Colección privada.

una palma (hoja de la palmera), término que se emplea especialmente para designar la que tiene sus lacinias (hojuelas) juntas y de color amarillo. Una de las más utilizadas en Europa es la palma de Canarias (Phoenix canariensis). Sin embargo, también se usa el término palma para las hojas de Cycas revoluta (Cycadaceae). El grabado de ramas de mirto y una palma, entrelazadas o no, al pie del escudo del país, no es exclusivo de las monedas de Costa Rica. En la Fig. 10B, se ilustra el anverso de un peso (8R) peruano, con los dos tipos de plantas. La Fig. 10 incluye el anverso (C) y reverso (D) de la moneda inglesa de seis peniques de 1840, que tiene grabadas una rama de mirto y otra que se asemeja a la hoja de una especie de encina, notándose en esta última las bellotas características del género Quercus. Ambas monedas circulaban en Costa Rica cuando se acuñaron (1842) las piezas de plata con la figura del tabaco (Fig. 5A), así como la figura de la ceiba (Fig. 4B) en el escudo de oro cuyo anverso, que incluye una palma y una rama de mirto, se ilustra en la Fig. 10A. Es nuestra opi- 


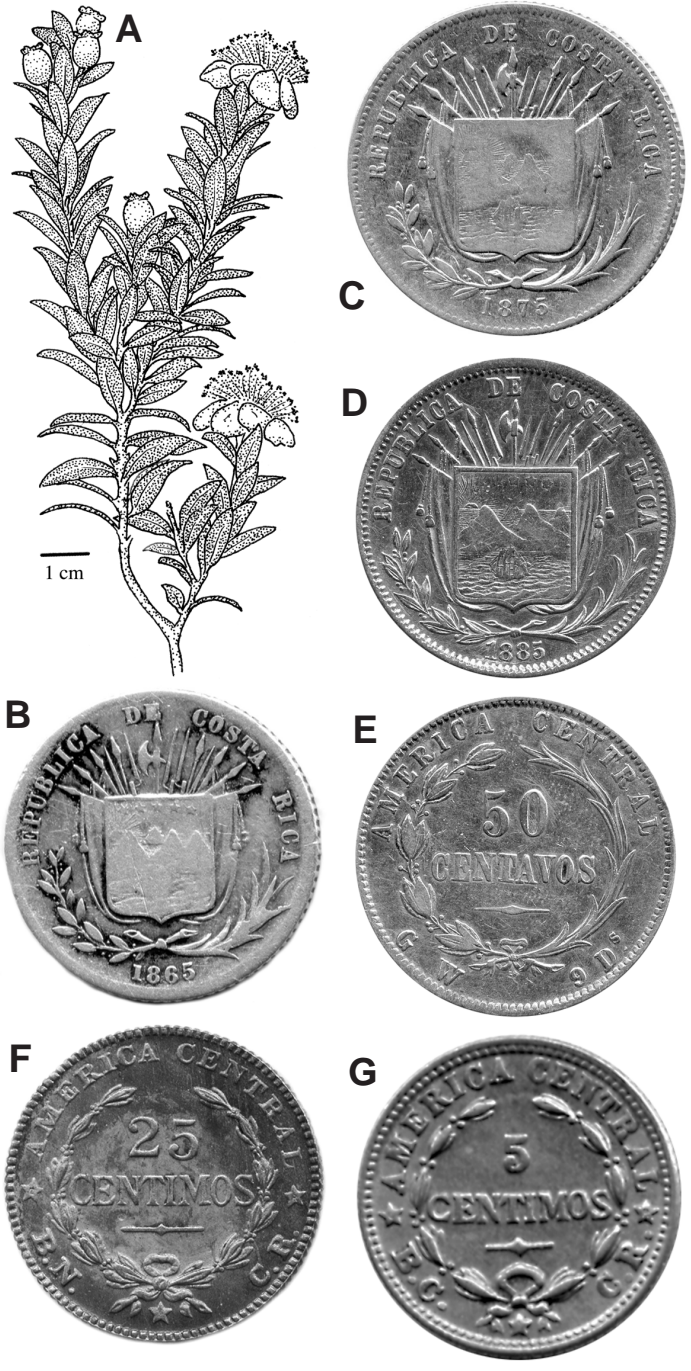

Figura 11. A. Rama del árbol Myrtus communis, basado en un ejemplar vivo que crece en un jardín en Santo Domingo, Heredia. Nótese los grupos de hojas elípticas, con nervadura central conspicua, frutos ovoides y flores con múltiples estambres. B,C,D,E y $\mathbf{F}$ : monedas con diferentes formas de representar al mirto y a la palma. B. Diez centavos, plata, San José, 1865, 19 mm /2.5 g. C. Cincuenta centavos, plata, San José, 1875, 33 mm /12.5 g. D,E . Cincuenta centavos, plata, San José, 1885, $31 \mathrm{~mm} / 12.5 \mathrm{~g}$. F. Veinticinco céntimos, latón, San José, 1946, 23 mm /3.5 g. G. Cinco céntimos, cuproníquel, Estados Unidos, 1951, 15 mm /1 g. (última moneda en que se ilustró al mirto).

nión que el modelo para la palma y el mirto en las monedas de Costa Rica estuvo inspirado en los pesos peruanos y mexicanos, así como en los peniques y chelines ingleses que circularon en el país y fueron contramarcadas en 1841 con la estrella de seis puntas o en 1850 con el león pasante (Fig. 10C), respectivamente. No hemos encontrado información que nos permita descartar la hipótesis de que la inclusión del mirto y del Quercus sp. (encina) para las monedas decretadas en 1848 (Fig. 6A,B) fue en parte motivado para hacer esas monedas más familiares en el comercio internacional, especialmente con el importante intercambio de la época con la Gran Bretaña. La similitud entre las hojas de varias especies de palmeras hace difícil el asignar las ilustradas en las monedas (Figs. 11B,C,D,E) a alguna o algunas especies conocidas. Sin embargo, no podemos excluir que a partir de las monedas de don Braulio Carrillo (Figs. 4C, 5A, 10A) se utilizara como modelo alguna palma de $A$. rostrata. En estas y otras monedas de Costa Rica una de las ramas coincide con algunas características de la especie Myrtus communis (Myrtaceae), mirto, llamado arrayán en otros países, por presentar hojas pequeñas, elípticas, opuestas, de borde liso, casi sésiles, con nervadura central conspicua y frutillos (bayas) globulares pedunculados. El mirto europeo (M. communis) es un arbusto de unos tres metros de altura, oloroso, con hojas duras, lustrosas y persistentes, flores blancas axilares, pequeñas, con muchos estambres cortos (Fig. 11A). El decreto emitido en 1848 especifica que la moneda de la República se sellará en el anverso con el escudo de armas descrito en el mismo decreto y en el cual también se indica que cerrarán el escudo dos palmas de mirto (Villalobos 1945). En la moneda de plata (Fig. 6A) se ilustra el escudo con armas y ramas de dos especies diferentes (mirto y palma, según nuestro criterio). Sin embargo, en la moneda de oro (Fig. 6B) se incluye otro modelo de escudo, sin armas y dos ramas de mirto bajo el escudo. Al menos ya en 1848 se utilizaba el término mirto en la descripción del escudo nacional y podría, por lo tanto, argumentarse que las dos ramas incluídas en esa moneda de oro y la rama de la derecha de esa moneda de plata corresponden, por coincidir con la forma de la planta de mirto, a la especie M. communis (mirto). El mirto fue y aún es un árbol importante en ritos religiosos y festejos antiguos (Lust 1990, Ciesla 2002). Es símbolo de paz, de gloria, y de amor pues, como ya mencionamos, estaba consagrado a Venus.

Según Lines (1948) en el anverso de las monedas decretadas en 1848 por el Dr. Castro (Figs. 6A,B) se 
anota que el Escudo de la República esté rodeado de palmas de mirto y éste autor opina que en las monedas de oro las palmas parecen ser sólo de café y en las de plata, de café y mirto. Según lo indicamos, es nuestro criterio que en la moneda de oro las dos ramas corresponden al mirto. Las ramas semejantes al M. соттиnis continúan utilizándose en varias monedas de Costa Rica del siglo XIX y hasta mitad del siglo XX (Fig. 11). Nótese que no obstante dos bandolas de café fueron grabadas en la moneda de 1935, el grabador utilizó el mirto en las pesetas de 1944, 1945 y 1946 (Fig. $11 \mathrm{~F})$, posiblemente debido a los problemas que aquejaban a la Casa de Moneda (Murillo 2004). El mirto también fue incluído por última vez en la moneda (Fig. 11G) de 5 céntimos (Troquel tipo I) de 1951 (Gurdián Montealegre 1997). En las Figs. 6, 10 y 11, también se puede notar los cambios en las representaciones del mirto y de la palma. Es posible que debido a que los nombres científicos de las especies de las dos ramas no se indican en los decretos respectivos, el grabador fue flexible en su escogencia de modelos, especialmente cuando se trata de acomodar en forma elegante las dos ramas en el estrecho espacio disponible. Esta flexibilidad artística es evidente aun en las representaciones de los escudos nacionales de Costa Rica ilustrados en sellos postales y timbres fiscales (Valverde Alpízar 2000) y en monedas; por ejemplo, las dos versiones del escudo nacional en la emisión resultante del decreto de 1848 (Fig. 6A,B). En el reciente libro Símbolos Nacionales de Costa Rica (Pacheco Ureña 2004), permanece sin abordar los temas de la identidad y el significado de las plantas representadas al pie de los escudos nacionales, desde 1824 al presente.

En la descripción de los escudos nacionales de 1848, 1906 y 1964 se especifica que, cerrarán el escudo dos palmas de mirto cubiertos con listón ancho que las une el cual será blanco y contendrá en letras de oro esta leyenda: República de Costa Rica (Villablobos 1945). Según lo indicamos anteriormente el término palma se utiliza para designar a la hoja de la palmera. Por tal motivo la expresión dos palmas de mirto resulta confusa para el botánico. El mirto es en el Escudo de Costa Rica emblema de solidaridad y victoria. Se incluyen dos ramas pues el triunfo se busca en dos ámbitos, el espiritual y el material (Altamirano 1997). No obstante esos decretos, la flexibilidad en los grabados de los escudos nacionales hizo que el mirto se incluyera cerrando (Fig. 12A) el escudo como lo indican los decretos, hasta 1935 , en las monedas de un colón, 25 y 50 céntimos.

En 1863, con la adopción del sistema decimal (vigente hasta 1896) se sustituyó la imagen de la India Parada en las monedas de oro (Fig. 6B), por la del valor correspondiente de la pieza colocado entre una guirnalda de laureles (Gurdián Montealegre 1997). En la Fig. 13 se incluye el anverso (A) y el reverso (B) de la moneda de oro de 10 pesos con las guirnaldas, que en este caso se asemejan mucho más a las hechas de mirto. El decreto de 1863 también incluye la emisión, por primera vez de piezas de un centavo (Fig. 13C,D) hechas de una aleación de cobre y níquel, las cuales por el reverso tienen indicado su valor entre una guirnalda de laureles. Para la moneda de 2 céntimos de 1903 (Fig. 5D,E) se especificó que en la parte inferior se incluya una rama de café entrelazada con otra de laurel (Gurdián Montealegre 1996). Sin embargo, en esa moneda la rama de la derecha es más afín a una de mirto que a una de laurel. En las monedas de un centavo de 1868 (Fig. 13C) las dos ramas son
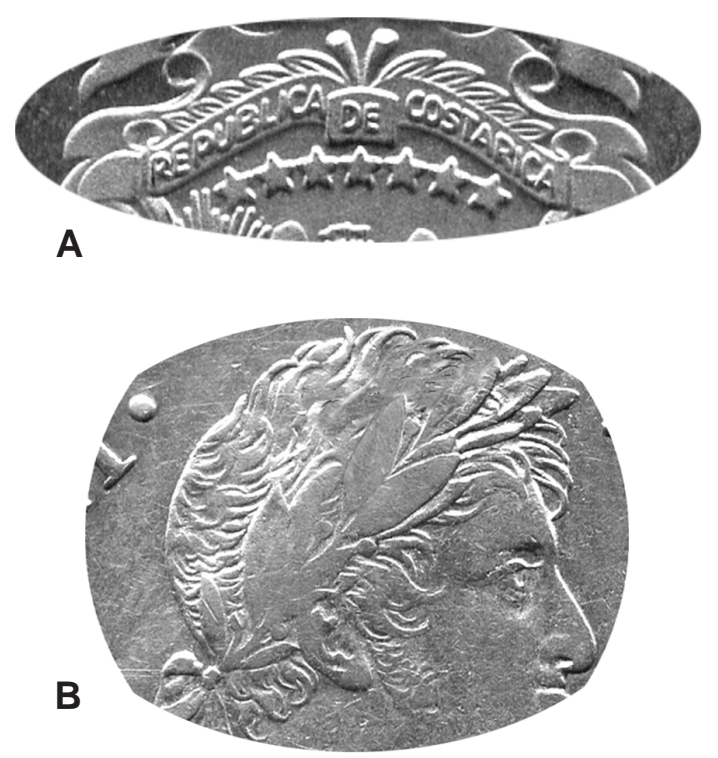

Figura 12. A. Detalle del anverso de la moneda de diez colones (Fig. 7A) mostrando dos ramas de mirto medio cubiertas y cerrando el Escudo Nacional. Nótese las nervaduras típicas. B. Detalle de la moneda de 8 Reales (Fig. 2A) mostrando la corona de laurel del rey Fernando VII. 

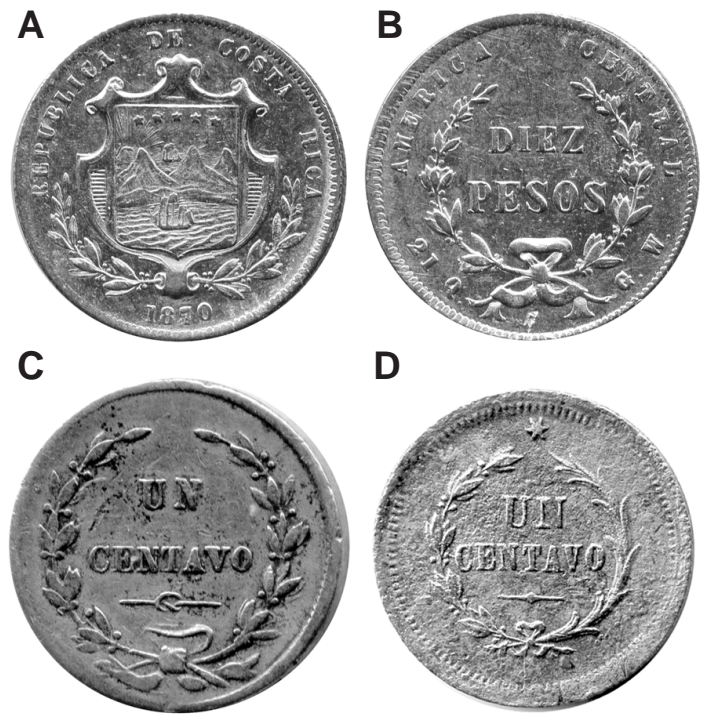

D

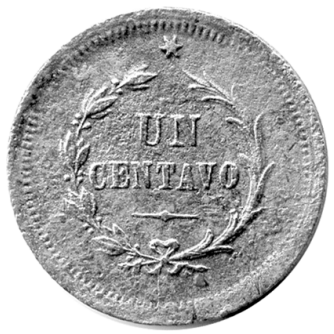

Figura 13. Diez pesos, oro, anverso (A) y reverso (B), San José, 1870, 29 mm /14.6 g. Colección privada. C. Un centavo, aleación níquel-cobre, San José, 1868, 20 mm /3.8 g. D. Un centavo, aleación níquel-cobre, San José, 1874, 20 $\mathrm{mm} / 3.8 \mathrm{~g}$. Colección privada.

también más similares al mirto que al laurel. En el centavo de 1874 (Fig. 13D) la rama de la izquierda presenta un mirto semejante al de otras monedas, como las incluidas en la peseta de 1946 (Fig. 11F), así como, a la derecha, una de la formas de representar a la palma. Es posible que el grabador de la Casa de Moneda utilizó el mismo modelo de mirto, de otras monedas de Costa Rica, en aquellas en que se requería ilustrar un laurel, aun en las que se acuñaron fuera de Costa Rica, suponemos siguiendo un modelo sugerido por la Casa de Moneda. Esto podría haber sucedido en virtud de la similitud que tendrían las ramas de las dos especies grabadas sin un objeto que proporcione una idea de su tamaño relativo. Además, ambas especies poseen frutillos globosos pedunculados y hojas de formas elípticas, lo cual induce a confusión. Siguiendo este argumento, el busto del rey Fernando VII (Figs. 2A y 12B) aparece ceñido con una corona de laurel, ya que el tamaño de las hojas es mucho más grande que el del mirto (Fig. 11A). El laurel europeo, consagrado a Apolo, es Laurus nobilis (Lauraceae), un árbol siempre verde de hasta siete metros de altura. Posee hojas coriáceas, persistentes, con pecíolo pequeño, oblongas, en menor número por eje que en el mirto y con frutos (bayas) de color negruzco. El laurel ha sido representado en América Central en otras piezas de interés numismático, como en las medallas acuñadas en 1921 en conmemoración del centenario de la independencia (Robinson 1965). En la Fig. 14 se incluye el anverso (A) y reverso (B) de una de esas medallas en la cual las hojas, frutos y ramas $(\mathrm{C})$, son semejantes a las de un ejemplar de L. nobilis. En las medallas de Costa Rica, acuñadas también para esa ocasión, las ramas ilustradas (Murillo 2004, p. 124) se asemejan al mirto de las monedas en la Fig. 11F,G. L. nobilis y M. communis no son especies nativas de Costa Rica. En el país se le llama laurel al árbol Cordia alliodora (Boraginaceae) y mirto a la especie Murraya paniculata (Rutaceae). Esta última, al igual que el M. communis, fueron introducidas a Costa Rica a mediados del siglo XIX (León y Poveda 2000).

En resumen, las monedas de Costa Rica incluyen una variedad de ilustraciones de plantas, lo que las hace particularmente atractivas para los coleccionistas especializados. Las monedas del árbol de encina y la india parada, acuñadas en la Casa de Moneda de San José, son en nuestra opinión las más logradas artísticamente, tanto por el detalle de la ilustración, como por el diseño de las piezas, no obstante las limitaciones de la época. En el siglo XXI, las monedas de arbolito destacan como un ejemplo de originalidad y de interés en

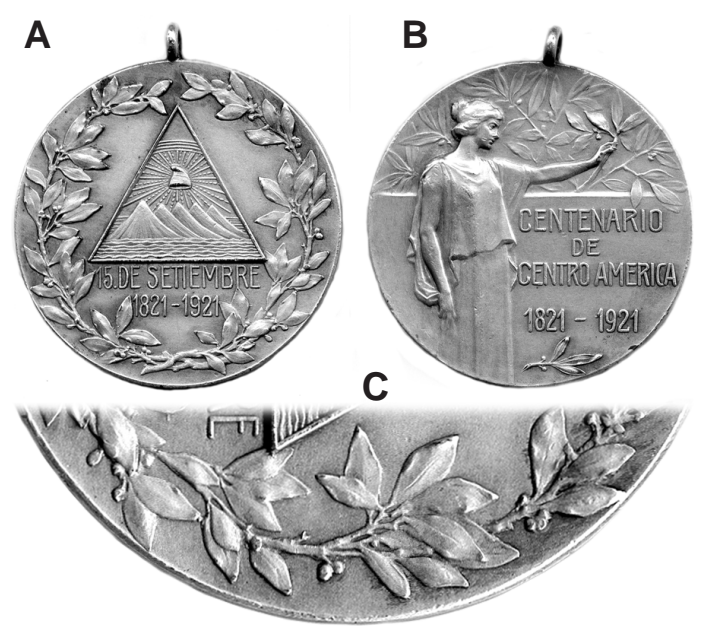

Figura 14. Anverso (A) y reverso (B) de una medalla conmemorativa del centenario de la independencia de Centroamérica. 1821-1921, plata, 36 mm / 16.8 g. C. Detalle de la rama de laurel del anverso. Nótese forma de la hoja, frutos y ramificaciones semejantes a $L$. nobilis. 
la naturaleza en el siglo XIX. En el siglo XX, ese ejemplo ha sido revivido a través de la emisión de varias monedas conmemorativas que incluyen, entre otras, las dedicadas a los símbolos nacionales como la guaria morada y el árbol de guanacaste.

Agradecimientos: A Jenaro Acuña, Andrea Bernecker, Minor Martin, y Karina Rodríguez por los comentarios. A Harlan K. Dean, Rodolfo Fernández, Jaime García, Jorge León y Axel Retana, por referencias de la literatura. A Manuel Chacón y a la Fundación Museos del Banco Central, Colección de Numismática, por las ilustraciones. Sergio Aguilar hizo la diagramación del texto y Jeffrey Sibaja dibujó el $M$. communis, basado en un ejemplar cultivado por Adriana Zamora. Este trabajo está dedicado a la memoria del Prof. José Antonio Vargas Salas (1916-2003), Maestro Normal (1938-1968), agricultor y artesano.

\section{LITERATURA CITADA}

Altamirano, C.L. 1997. Los Símbolos Nacionales de Costa Rica. 2da. Ed. Los Sauces. San José. 97 p.

Blanco Quirós, M. 1994. Historia de la Moneda. Anales de la Universidad de Costa Rica 8: 39-46.

Burger, W. 1977. Flora Costaricensis: Fagaceae. Fieldiana Botany 40: 59-82.

Carranza Astúa, J. A. 2001. Historia de los billetes de Costa Rica 1858-2001. Fundación Museos del Banco Central - Litografia e Imprenta Lil. San José. 231 p.

Chacón, M.B. 2000. Del Estado a la República: las monedas y la política (1821-1850). Banco Central de Costa Rica, San José.

Chacón Hidalgo, M.B. \& E. Carazo de Flores. 2002. Los boletos de café en Costa Rica. Folleto Técnico. Fundación Museos del Banco Central. 20 p.

Chacón Hidalgo, M.B. 2003. Monedas de Costa Rica: Reseña Histórica. Editorial de la Universidad de Costa Rica. San José. 87 p.

Chavarría L. 2003. Manojo de Guarias. Editorial UNED. San José. 52 p.

Ciesla, W.M. 2002. Non-wood forest products from temperate broad-leaved trees. Food and Agriculture Organization of the United Nations. Rome. $200 \mathrm{p}$.

Coste, R. 1968. El café. Editorial Blume. Barcelona. 285 p.

De Anglería, P. M. 1944. Décadas del Nuevo Mundo. Bajel. Buenos Aires. 673 p.

Dueñas Leiva. T. 1994. Monedas Tipo de Costa Rica. Editorial UNED, San José. 71 p.

Dressler, R.L. \& W.E. Higgins. 2003. Guarianthe, generic name for the "Cattleya" skinneri complex. Lankesteriana $7: 37-38$.

Fallas Barrantes, M.A. 1967. Una institución colonial: la Factoría de Tabacos de Costa Rica. Tesis de Licenciatura. Universidad de Costa Rica. 333 p.

Fernández de Oviedo, G. 1944. Historia General y Natural de las Indias. Vol. III. Guaranía, Asunción. 319 p.
Fernández Guardia, R. 1980. Cosas y Gentes de Antaño. Editorial UNED. San José. 250 p. 2002. Costa Rica en el Siglo XIX: Antología de Viajeros. Editorial UNED. San José. 495 p.

Font Quer, P. 1973. Diccionario de Botánica. Editorial Labor, Madrid. 1244 p.

González Zeledón, M. 1998. Cuentos de Magón. Editorial Costa Rica. San José. 403 p.

Green, P.M. 2003. Rumors of great rarities make the rounds. World Coin News. 12: 58-60.

Gurdián Montealegre, R. 1997. Contribución al estudio de las monedas de Costa Rica. Segunda Edición. Litografía e Imprenta Lil. San José. 186 p.

Hammel, B.E., M.H. Grayun, C. Herrera y N. Zamora (Eds). 2003. Manual de Plantas de Costa Rica. Vol.II. Gimnospermas y Monocotiledóneas. Monographs in Systematic Botany from the Missouri Botanical Garden 92: 1-693.

Krause, C.L. \& C. Mishler. 2001. Standard Catalogue of World Coins 1801-1900. Krause Publications, Iola. 1200 p. 2004 Standard Catlaogue of World Coins 1901- Present. Krause Publications, Iola. 2224 p.

León, J. \& L.J. Poveda. 2000. Los nombres comunes de las plantas de Costa Rica. Editorial Guayacán. San José. 870 p.

Lines, J.A. 1946. Libre Crezca Fecundo. Revista del Banco Nacional de Seguros 5: 7-17.

1948. Las monedas de oro y plata emitidas por el Dr. Castro. Revista de los Archivos Nacionales 9/10: 505-513.

Lust, J. 1990. The herb book. Bantam. New York. 659 p.

Melliss, E. 1891. Informe sobre Las Minas del Aguacate y de Los Castros. Instituto físico-geográfico nacional de Costa Rica. Tipografía Nacional. San José. 28 p.

Murillo, J. 2004. Historia de las Monedas de Costa Rica. Catálogo Numismático. Editorial UNED. San José. 252 p.

Pacheco Ureña A.P. 2004. Símbolos Nacionales de Costa Rica. Imprenta Nacional. San José. 348 p.

Robinson, C.M. 1965. The coins of Central America 17331965. An illustrated guide. C.M. Robinson. San Benito. $131 \mathrm{p}$.

Rojas, F. 1993. Arboles Nacionales. Editorial Tecnológica de Costa Rica. Cartago. 98 p.

Soto, R. \& D.L. Ballantine. 1986. La flora bentónica marina del Caribe de Costa Rica. Brenesia 25/26: 123-162.

Valerio, J. 1962. La moneda de cacao: Turrialba recobra importancia. Educación (MEP) 30/31: 35-36.

Valverde Alpízar, S.A. 2000. El Escudo Nacional de Costa Rica. Análisis Heráldico. Revista de la Academia Costarricense de Ciencias Genealógicas 38: 477-494.

Vega Jiménez, P. 1991. De la banca al sofá: la diversifica- 
ción de los patrones de consumo en San José (1857- Villalobos Rodríguez, J.H., L.A. Chacón \& J.F. Saénz1861). Revista de Historia 24: 53-85.

Carbonell. 2000. Braulio Carrillo: El Estadista. Tomo

Villalobos, D. 1945. Banderas y Escudos de Costa Rica.

II. Imprenta Nacional. San José. 624 p.

Educación 139: 18-23. 